\title{
Improving data quality for 3D electron diffraction (3DED) by Gatan Image Filter Taimin Yang ${ }^{1}$, Hongyi $\mathrm{Xu}^{2}$, Xiaodong Zou ${ }^{3}$ \\ ${ }^{1}$ Stockholm University ${ }^{2}$ Department of Materials and Environmental Chemistry, ${ }^{3}$ Stockholm University \\ taimin.yang@mmk.su.se
}

\begin{abstract}
Electrons interact with matter 1e6 times stronger than X-rays do, which makes it an ideal radiation source for diffraction and imaging experiments on submicron- and nano-sized crystals. During the last three decades, 3D electron diffraction (3D ED) has been developed into a regular and reliable technique for structure determination, which is complementary to single-crystal X-ray diffraction (SCXD) and single particle analysis. One issue for electron diffraction is inelastic scattering, which brings background in the diffraction patterns. This background is most obvious for electron diffraction patterns from protein crystals, especially at low angles. Even though modern diffraction data software (XDS, DIALS, MOSFLM) has sophisticated background removal algorithms to deal with this, the existence of inelastic scattering will still add errors in the diffraction experiment. The inelastically scattered electrons can be removed by energy filters. Here, we implemented energy-filtered 3D ED using a Gatan Energy Filter (GIF) in both TEM selected area electron diffraction mode and STEM micro/nanoprobe mode. We explained the setup in detail and this implementation can allow researcher to have better accessibility to energy-filtered 3DED experiments because more microscopes are equipped with a GIF than an in-column omega filter. We also proposed a crystal tracking method in STEM mode using live HAADF image stream. This method enables us to collect energy-filtered 3DED datasets in STEM mode with a larger tilt range without foregoing any frames. This can avoid crystal moving out of the beam during the tilting and the tilt range can always reach the maximum tilt range of the microscope (in our case $\sim 150^{\circ}$ ). We acquired multiple datasets from different crystals and we further processed and refined the structures. The obtained structure after refinement also improved. Hydrogen atoms around the nitrogen atoms were found in $\mathrm{NH} 4 \mathrm{H} 2 \mathrm{PO} 4$ crystals and the atomic displacement parameters were physically sensible for energy-filtered datasets. We observed that the final R1 will improve $20 \%$ to $30 \%$ for energy-filtered datasets compared with unfiltered datasets. We also discussed the possible reasons that lead to the improvement.
\end{abstract}

